\title{
SPATIALLY SELECTIVE MODIFICATION OF GOLD NANORODS
}

\author{
1,2Anastasiia OLSHTREM, 1,2Olga GUSELNIKOVA, ${ }^{2}$ Pavel POSTNIKOV, ${ }^{1}$ Vaclav SVORCÍK, \\ 1,2Oleksiy LYUTAKOV
}

1 University of Chemistry and Technology, Prague, Czech Republic, EU, Iyutakoo@vscht.cz

${ }^{2}$ Tomsk Polytechnic University, Research School of Chemistry and Applied Biomedical Sciences, Tomsk, Russian Federation

https://doi.org/10.37904/nanocon.2019.8614

\begin{abstract}
In this contribution, we present the new approach for plasmon assisted grafting of anisotropic gold nanorods (AuNRs) with spatial selectivity, which was used for creation of nanoparticles with amphiphilic surface. The surface modification was carried out in two stages with implementation of: (I) the plasmon-activated iodonium salt (IS), and (II) the aryl diazonium salt (ADT). The plasmon assisted activation of IS was performed under the illumination of AuNRs with wavelength corresponded to the longitudinal plasmon resonance of AuNRs and resulted in grafting of organic moieties (Ar-(CF3)2) to AuNRs edges. The AuNRs lateral sides remains ungrafted and were further decorated with ADT-COOH. As result of varying substituents in the chemical structures of iodonium and diazonium salts the amphiphilic NPs with spatially divided hydrophobic (grafted to AuNRs edges) and hydrophilic (grafted to AuNRs lateral sides) chemical groups were obtained. Corresponded UV-Vis and Raman measurements confirm the success of AuNRs chemical grafting. As result, the AuNRs with spatially separated chemical moieties were created in very simple way, primarily in solution and without the need of sophisticated technique of NPs mobilization or their surface screening.
\end{abstract}

Keywords: Plasmon-assisted chemical transformation, gold nanorods, surface grafting, amphiphilic nanoparticles

\section{INTRODUCTION}

In recent years, gold and silver nanoparticles (metal nanoparticles - MeNPs) have been used as effective optical transducers for chemical and biochemical sensing in many areas of medicine and safety [1-10]. Of particular interest is the potential spatial selective introduction of chemical moieties on MeNPs surfaces for potential design of new elements for monitoring and removal / separation of microorganisms and cells [11]. For example, recently developed nanoparticle-based materials for cancer therapies can be mentioned [12].

Gold nanomaterials, especially gold nanorods (AuNRs), attract much scientific attention. First, the effective excitation of surface plasmon resonance (SPR) on AuNRs makes it possible to effectively absorb light even with a small amount of nanoparticles [13,14]. Second, the SPR absorption band of AuNRs can easily be moved into the near infrared spectral region (NIR) where light has a "biological" transmission window $[15,16]$. Finally, AuNRs can be readily modified with organic compounds by thiol Au-S and covalent bonds [17-19]. However, the uncontrolled and unstable process of modifying the surface of nanoparticles can be a serious problem when using nanoparticles in (bio)environment. Especially the non-covalent nature of the commonly used Au$S$ interaction may cause some instability of grafted functional groups.

In this contribution, we propose the new approach for spatially selective plasmon-controlled surface modifications of AuNRs. The modification was carried out with using of iodonium and diazonium chemistry and nanoparticles with amphiphilic surface were obtained. The resulting surface modified AuNRs can be used in next generation of biochemical and molecular genetic diagnostic systems. 


\section{EXPERIMENTAL}

\subsection{Materials}

Chloroauric acid tetrahydrate $(99.9 \%)$, silver nitrate $(99 \%)$, ascorbic acid (99 \%), hexadecyltrimethylammonium bromide ( $\geq 98 \%)$, sodium borohydride ( $\geq 99 \%)$, copper (II) chloride (99\%), 3,5Bis(trifluoromethyl)phenyl-boronic acid ( $\geq 95 \%)$, 1-iodo-3,5-bis(trifluoromethyl)benzene (98 \%), boron trifluoride diethyl etherate (99\%), 3-Chloroperbenzoic acid ( $\leq 77 \%)$, 4-aminobenzoic acid (ReagentPlus, $\geq 99 \%$ ), tert-Butyl nitrite (90\%), acetic acid (reagent grade, $\geq 99.7 \%$ ), diethyl ether, methanol and Toluenesulfonic acid monohydrate $(\geq 98.5 \%)$ were purchased from Sigma-Aldrich. All reagents were used without further purification. Throughout the all experiments deionized water was used. 4carboxybenzenediazonium tosylate was prepared according to [20]. Di-[3,5-bis(trifluoromethyl)phenyl] iodonium tetrafluoroborate was prepared according to [21].

\subsection{Samples preparation}

AuNRs were prepared by the slightly modified method described in [22]. The seeds were prepared by reduction of $\mathrm{CuCl} 2$ solution ( $500 \mu \mathrm{l}$ of $10 \mathrm{ppm}$ ) with freshly prepared ice-cold NaBH4 $(100 \mu \mathrm{l}$ of $10 \mathrm{mM})$. The reagents were loadings is as follows: $10 \mathrm{ml}$ of $100 \mathrm{mM} \mathrm{CTAB}, 500 \mu \mathrm{l}$ of $10 \mathrm{mM} \mathrm{HAuCl}{ }^{*} 3 \mathrm{H} 2 \mathrm{O}, 65 \mu \mathrm{l}$ of $10 \mathrm{mM} \mathrm{AgNO3}$, and $70 \mu \mathrm{l}$ of $100 \mathrm{mM} \mathrm{AA}$ and then $100 \mu \mathrm{l}$ of the Cu seed particles. The solution was kept undisturbed for $3 \mathrm{~h}$ at room temperature. Then the solution temperature was decreased to $8^{\circ} \mathrm{C}$ for $1 \mathrm{~h}$. The obtained AuNRs were purified using the subsequent cycles $(3 \mathrm{x})$ of centrifugation and ultrasound-assisted re-dispersion.

\section{Plasmon assisted iodonium surface grafting}

To the solution of IS ( $1 \mathrm{ml}$ of $1 \mathrm{mM}$ water/methanol solution (9:1, respectively) was added the colloid suspension of gold nanorods $(2 \mathrm{ml})$ under stirring and illuminated with LED light under the continuous mixing for $2 \mathrm{~h}$. Laser triggering was performed using the $785 \mathrm{~nm}(\mathrm{~mW})$ continuous laser source. The laser beam spot (ca $5 \mathrm{~cm} 2$ in area) was set up to homogeneously illuminate the reaction mixture. After the modification process, AuNRs were collected by centrifugation and washed sequentially by water and a water/methanol mixture (1:1).

\section{Spontaneous diazonium surface grafting}

To suspension of IS grafted AuNRs was added an aqueous solution of the 4-carboxybenzenediazonium (1 ml of $1 \mathrm{mM}$ ) and stirred for $2 \mathrm{~h}$ in dark. The nanoparticles were then collected by centrifugation and washed thoroughly by several centrifugation/washing cycles by water and a water/methanol mixture.

\subsection{Measurement techniques}

For the characterization of the solutions, the UV/Vis/NIR spectroscopy technique was used in the spectral range $300-900 \mathrm{~nm}$, scanning rate $480 \mathrm{~nm} \mathrm{~min}^{-1}$, data collection interval $1 \mathrm{~nm}$. The solutions were measured in $1 \mathrm{~cm}$ quartz cells.

TEM images were obtained on a JEOL JEM-1010 transmission electron microscope with a SIS MegaView III digital camera, STEM observations and EDX analyses were performed on a JEOL JEM-2200FS electron microscope equipped with a $100 \mathrm{~mm} 2$ JEOL Dry SD100GV EDX detector with a Super UTW window.

Raman spectra were measured on ProRaman-L (Laser power $30 \mathrm{~mW}$ ) Raman spectrometer with $785 \mathrm{~nm}$ excitation wavelengths. Spectra were measured 50 times, each of them with $5 \mathrm{~s}$ accumulation time.

\section{RESULTS AND DISCUSSION}

Recently anisotropic nanoparticles in the form of rods attracted particular attention due to their unique optical properties. The absorption spectrum of such nanoparticles contains two SPR bands (in the visible and near- 
infrared regions) related to dipoles oscillating along and across the axis of the cylinder, which corresponds to the longitudinal and transverse plasmon resonances, respectively. The practical utilization of nanorods requires their surface functionalization to protect against aggregation, as well as to control the optical, electronic, and catalytic properties. In our experiments nanorods were obtained by a method described in [22]. The resulting solutions had a dark purple color. The obtained nanoparticles were characterized by UV-type spectroscopy and TEM analysis (Figure 1).

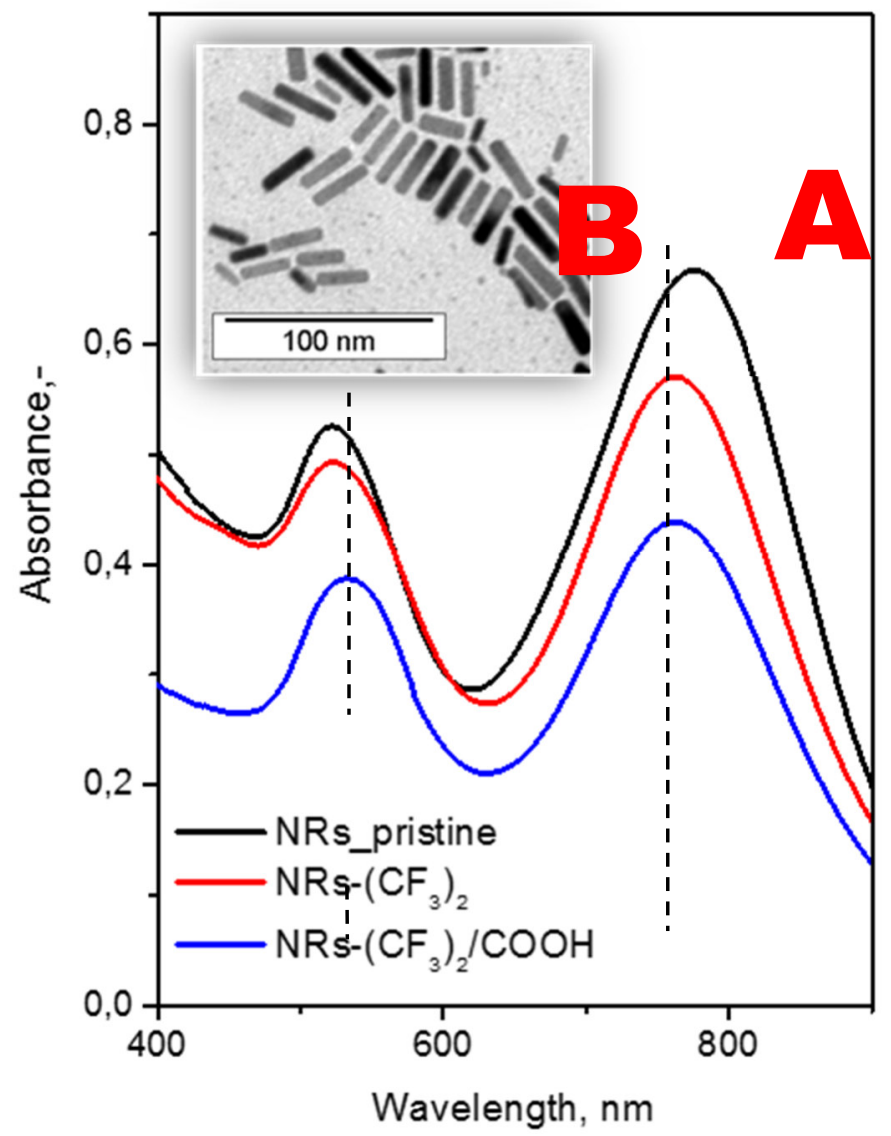

Figure 1 (A) - UV-Vis spectra of pristine AuNRs, AuNRs after the plasmon assisted grafting of IS, and AuNRs after the diazonium-based grafting of AuNRs lateral sides; (B) - TEM images of pristine AuNRs, involved in further experiments

From Figure 1B it can be seen that the applied technique really leads to the formation of cylindrical nanoparticles, the length of which is $40-50 \mathrm{~nm}$. The resulting nanorods have two absorption peaks at 532 and $780 \mathrm{~nm}$, which corresponds to transverse and longitudinal plasmons, respectively (Figure 1A). Surface modification of IS - di-[3,5-bis(trifluoromethyl)phenyl] iodonium tetrafluoroborate - IS - was carried out by adding a water-alcohol solution of salt to a suspension of nanorods under a Raman laser with a wavelength of $785 \mathrm{~nm}$. The advantage of the ISs is its a relatively high reduction potential, which results in these salts being practically not susceptible to spontaneous binding to the surface, but can be activated by plasmon triggering, allowing speed control and spatial control of grafting. In addition, which, as you know, iodonium salts are a source of highly active types of aryl radicals. The main concept of modification is based on plasmon activation of IS under the illumination at the wavelengths corresponding to the longitudinal plasmon resonance band and interaction of created radicals with spatially-closed nanorods edges. Thus, organic moieties are grafted only on the side surfaces of nanoparticles, in the site of plasmonic hot spots excitation (schematic representation of process is given in Figure 2). 


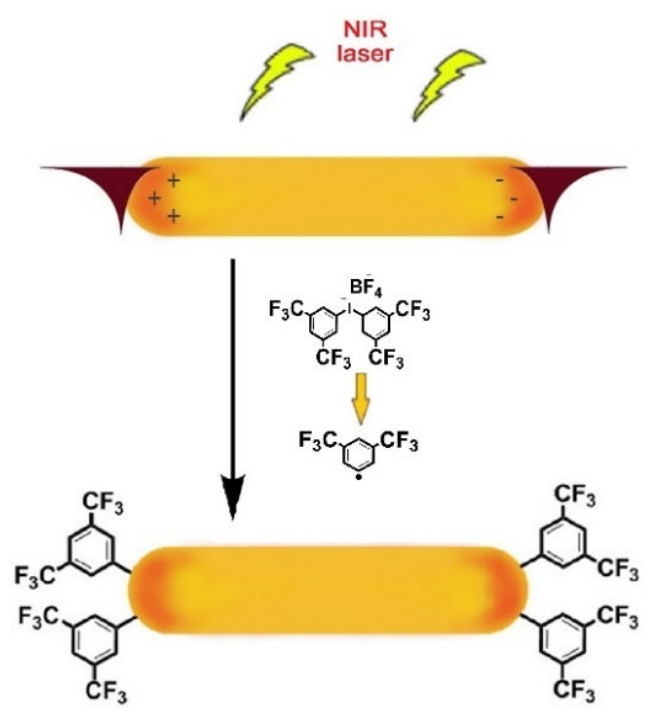

Figure 2 Scheme for obtaining surface modified gold nanophores using IS in wavelength laser irradiation, activation of longitudinal surface

After modification, a plasmon shift of the corresponding longitudinal section occurs (Figure 1A, red spectrum)., which confirms the activation of the longitudinal surface at a wavelength of $785 \mathrm{~nm}$, while the lateral side remains unscreened and can furthers be used for second step of surface modification by spontaneous diazonium grafting After the functionalization of the nanoparticles with ADT in the spectrum, we can observe a shift in the absorption peak corresponding to the transverse plasmon (Figure 1A, blue spectrum).

The two steps of AuNRs grafting procedures were checked also using the surface enhanced Raman spectroscopy (SERS) measurements (Figure 3).

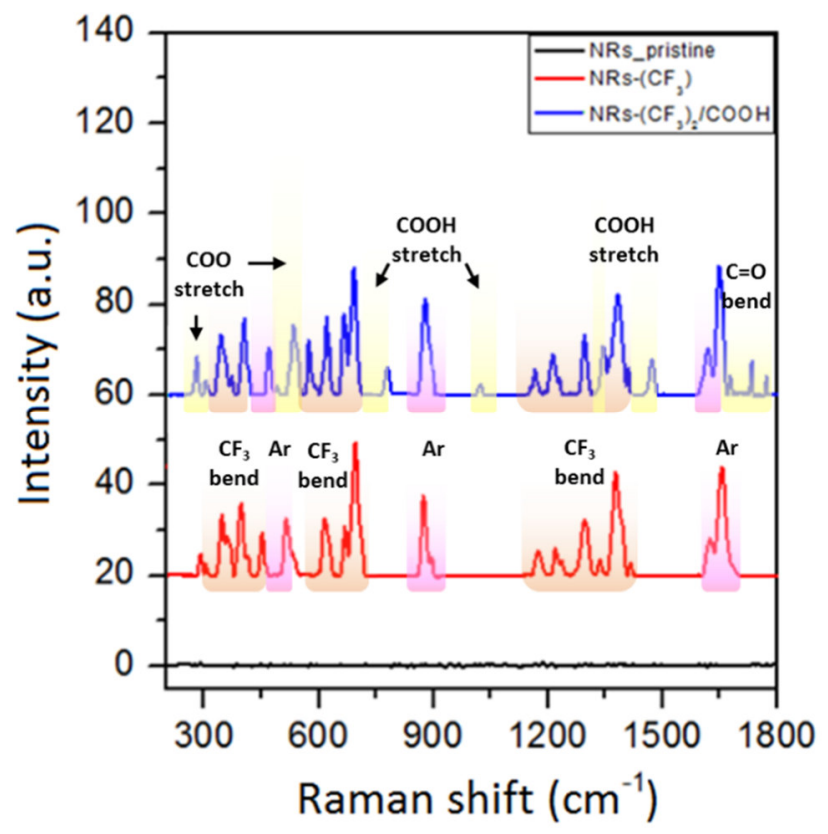

Figure 3 SERS spectra, measured on AuNRs grafted with $\operatorname{Ar}-\left(\mathrm{CF}_{3}\right)_{2}$ (AuNRs edges - red spectrum) and $\mathrm{Ar}-\mathrm{COOH}$ (AuNRs lateral sides - blue spectrum) 
The spectra were adjusted along the baseline by subtracting the SERS spectrum from the primary AuNRs and removing the uninformative signal from the absorbed CTAB. After the grafting procedure, the Raman bands, typical for $\mathrm{Ar}-(\mathrm{CF} 3)_{2}$ and $\mathrm{Ar}-\mathrm{COOH}$ become apparent (characteristic bands are designated by arrows). The results of SERS confirm a spatially selective grafting.

\section{CONCLUSION}

In this work, we present a new method of spatially selective plasmon-activated modification of anisotropic AuNRs to produce an amphiphilic surface. Activation of the longitudinal surface by incident light led to grafting of organic fragments $\left(\mathrm{Ar}-\left(\mathrm{CF}_{3}\right)_{2}\right)$ to the edges of AuNR. The remaining transverse surfaces were then modified spontaneously with the diazonium salt of ADT-COOH. As a result, AuNPs with spatially separated hydrophobic (grafted to AuNR edges) and hydrophilic (grafted to the lateral sides of AuNR) chemical groups were obtained. Proposed approach was realized in solution and does not require the surface assistance in the spatially selective modification of AuNRs. The resulting surface modified gold nanoparticles can be used to create a next generation of biochemical and molecular genetic diagnostic systems.

\section{ACKNOWLEDGEMENTS}

This work was financially supported by the Internal Grant Agency UCT № 126881910.

\section{REFERENCES}

[1] WILEY, Benjamin, SUN, Yugang, MAYERS, Brian, XIA, Younan. Shape-controlled synthesis of metal nanostructures: the case of silver. Chem. Eur. J. 2005. 11, pp. 454-463.

[2] KIM, Franklin, CONNOR, Stephen, SONG, Hyunjoon, KUYKENDALL, Tevye, YANG, Peidong. Platonic gold nanocrystals. Angew. Chem. Int. Ed. 2004. 43, pp. 3673-3677.

[3] DEBRINA, Jana, MATTI, Carlos, HE, Jie, SAGLE, Laura. Capping agent-free gold nanostars show greatly increased versatility and sensitivity for biosensing. Anal. Chem. 2015. 87, pp. 3964-3972.

[4] MIESZAWSKA, Aneta J., MULDER, Willem J. M., FEYARD, Zahi A., CORMODE, David P. Multifunctional gold nanoparticles for diagnosis and therapy of disease. Mol. Pharm. 2013. 10, pp. 831-847.

[5] CHENG, Han-Wen, HUAN, Shuang-Yan, WU, Hai-Long, SHEN, Guo-Li, YU, Ru-Qin. Surface-Enhanced Raman Spectroscopic Detection of a Bacteria Biomarker Using Gold Nanoparticle Immobilized Substrates. Anal. Chem. 2009. 81, pp. 9902-9912.

[6] RAMAN, C.V., KRISHNAN, K.S. A New Type of Secondary Radiation. Nature. 1928. 121, pp. 501-502.

[7] BONHAM, Andrew J., BRAUN, Gary, PAVEL, Ioana, MOSKOVITS, Martin, REICH, Norbert O. Detection of Sequence-specific Protein-DNA Interactions via Surface Enhanced Resonance Raman Scattering. J. Am. Chem. Soc. 2007. 129, pp. 14572-14573.

[8] BRUS, Louis. Photochemistry and Single-Molecule Raman Spectroscopy. Acc. Chem. Res. 2008. 41, pp. 17421749 .

[9] KUMAR, G. V. Pavan, REDDY, B. A. Ashok, ARIF, Mohammed, KUNDU, Tapas K., NARAYANA, Chandrabhas. Surface-Enhanced Raman Scattering Studies of Human Transcriptional Coactivator p300. J. Phys. Chem. B. 2006. 110, pp. 16787-16792.

[10] CHEN, Lei, CAI, Linjun, RUAN, Weidong, ZHAO, Bing. Surface-enhanced Raman Spectroscopy (SERS): Protein Application. John Wiley \& Sons, Ltd. Encyclopedia of Analytical Chemistry. 2013.

[11] HAN, Xiao X., KITAHAMA, Yasutaka, ITOH, Tamitake, WANG, Chun X., ZHAO, Bing, OZAKI, Yukihiro. ProteinMediated Sandwich Strategy for Surface-Enhanced Raman Scattering: Application to Versatile Protein Detection. Anal. Chem. 2009. 81, pp. 3350-3355.

[12] AN, Lu, WANG, Yuanyuan, TIAN, Qiwei, YANG, Shiping. Recent Advances in Synthesis, Biological Imaging, and Cancer Therapy. Materials. 2017. 10, pp. 1372. 
[13] MACKEY, Megan A., ALI, Moustafa R. K., AUSTIN, Lauren A., NEAR, Rachel D., EL-SAYED, Mostafa A. The most effective gold nanorod size for plasmonic photothermal therapy: Theory and in vitro experiments. J. Phys. Chem. B. 2014. 118, pp. 1319-1326.

[14] YANG, Xuan, YANG, Miaoxin, PANG, Bo, VARA, Madeline, XIA, Younan. Gold nanomaterials at work in biomedicine. Chem. Rev. 2015. 115, pp. 10410-10488.

[15] BURROWS, Nathan D., HARVEY, Samantha, IDESIS, Fred A., MURPHY, Catherine J. Understanding the seedmediated growth of gold nanorods through a fractional factorial design of experiments. Langmuir. 2017. 33, pp. 1891-1907.

[16] ZHANG, Liming, XIA, Kai, LU, Zhuoxuan, LI, Guopeng, CHEN, Juan, DENG, Yan, LI, Song, ZHOU, Feimeng, HE, Nongyue. Efficient and facile synthesis of gold nanorods with finely tunable plasmonic peaks from visible to nearIR range. Chem. Mater. 2014. 26, pp. 1794-1798.

[17] ZHOU, Wen, GAO, Xia, LIU, Dingbin, CHEN, Xiaoyuan. Gold nanoparticles for in vitro diagnostics. Chem. Rev. 2015. 115, pp. 10575-10636.

[18] AHMAD, Randa, BOUBEKEUR-LECAQUE, Leïla, NGUYEN, Mai, LAU-TRUONG, Stephanie, LAMOURI, Aazdine, DECORSE, Philippe, GALTAYRIES, Anouk, PINSON, Jean, FELIDJ, Nordin, MANGENEY, Claire. Tailoring the surface chemistry of gold nanorods through Au-C/ Ag-C covalent bonds using aryl diazonium salts. J. Phys. Chem. C. 2014. 118, pp. 19098-19105.

[19] YOSHIMURA, Akira, ZHDANKIN, Viktor V. Advances in Synthetic Applications of Hypervalent lodine Compounds. Chem. Rev. 2016. 116, pp. 3428-3435.

[20] FILIMONOV, Victor D., TRUSOVA, Marina, POSTNIKOV, Pavel, KRASNOKUTSKAYA, Elena A., LEE, Young Min, HWANG, Ho Yun, KIM, Hyunuk, CHI, Ki-Whan. Unusually stable, versatile, and pure Arenediazonium Tosylates: their preparation, structures, and synthetic applicability. Org. Lett. 2008. 10, pp. 3961-3964.

[21] HE, Maggie, SWAGER, Timothy M. Covalent functionalization of carbon nanomaterials with iodonium salts. Chem. Mater. 2016. 28, pp. 8542-8549.

[22] SAMAL, Akshaya Kumar, SREEPRASAD, Theruvakkattil Sreenivasan, PRADEEP, Thalappil. Investigation of the role of $\mathrm{NaBH} 4$ in the chemical synthesis of gold nanorods. J. Nanopart. Res. 2010. 12, pp. 1777-1786. 